\title{
Effect of Herb-Partitioned Moxibustion on Autophagy and Immune-Associated Gene Expression Profiles in a Rat Model of Crohn's Disease
}

\author{
Ji-meng Zhao $\mathbb{D}^{1},{ }^{1}$ Ya-nan Liu, ${ }^{2}$ Han-dan Zheng, ${ }^{2}$ Yan Huang $\mathbb{D},{ }^{1}$ Qin Qi, ${ }^{2}$ Hui-rong Liu $\left(\mathbb{D},{ }^{1}\right.$ \\ Yin Shi $\mathbb{D}^{1},{ }^{1}$ Xiao-peng Ma $\mathbb{D}^{1},{ }^{1}$ Yuan Lu $\mathbb{D}^{1},{ }^{1}$ and Lu-yi Wu $\mathbb{D}^{3}$ \\ ${ }^{1}$ Key Laboratory of Acupuncture and Immunological Effects, Shanghai Research Institute of Acupuncture and Meridian, \\ Shanghai University of Traditional Chinese Medicine, Shanghai 200030, China \\ ${ }^{2}$ Shanghai University of Traditional Chinese Medicine, Shanghai 201203, China \\ ${ }^{3}$ Shanghai Qigong Research Institute, Shanghai University of Traditional Chinese Medicine, Shanghai 200030, China
}

Correspondence should be addressed to Yuan Lu; luyuan_sh@163.com and Lu-yi Wu; luyitcm@163.com

Received 24 October 2018; Revised 9 January 2019; Accepted 3 February 2019; Published 7 March 2019

Academic Editor: Luigi Milella

Copyright (c) 2019 Ji-meng Zhao et al. This is an open access article distributed under the Creative Commons Attribution License, which permits unrestricted use, distribution, and reproduction in any medium, provided the original work is properly cited.

Objective. To investigate the immune regulation mechanism of herb-partitioned moxibustion in rats with Crohn's disease (CD) focusing on autophagy. Methods. Rats were randomly divided into normal (N) group, CD model (M) group, CD model with herb-partitioned moxibustion (MM) group, normal with herb-partitioned moxibustion (NM) group, CD model with mesalazine (western medicine, Med ) group, and normal saline (NS) group, with 10 rats in each group. The CD model rats were prepared by trinitrobenzene sulphonic expect for the $\mathrm{N}$ group and NM group. After the CD rats model were established, the rats in the MM and NM groups were treated with herb-partitioned moxibustion at Tianshu (ST25) and Qihai (CV6) acupoints once daily for 7 days, and rats in the Med and NS groups were respectively treated with mesalazine enteric coated tablet and normal saline once daily for 7 days. After intervention, hematoxylin-eosin staining was used to observe the histological changes of colon; RNA sequencing was used to observe the changes in autophagy- and immune-associated gene expression profiles. In addition, autophagy- and immuneassociated cytokines and signaling pathways in CD rats were also screened. Results. HPM significantly increased the body weight of $\mathrm{CD}$ rats $(P<0.01)$ and improved the pathological injury of colon in $\mathrm{CD}$ rats $(P<0.01)$. HPM also changed the expression of many autophagy- and immune-associated genes, especially downregulating the expression of autophagy-associated Nod2, Irgm genes as well as the receptor of immune-associated Il12b, Il22 (Ill2rb1, Il22ra2) genes in the colon of CD rats. HPM also changed the enrichment levels of differentially expressed genes in the human T-cell leukemia virus type-1 infection pathway, the Epstein-Barr virus infection pathway, and the cell adhesion molecule pathway. In addition, the expression levels of Nod2, Irgm, IL-12b, and IL-22 mRNA were increased (all $P<0.01$ ) in the M group compared to the $\mathrm{N}$ group, while the expression levels of Nod2, Irgm, IL-12b, and IL-22 mRNA were decreased $(P<0.05$ or $P<0.01)$ in the MM and Med groups compared to the M group. Conclusion. Herbpartitioned moxibustion may effectively attenuate intestinal inflammation and promote the repair of colon mucosal injury of CD rats through the regulation of autophagy- and immune-associated gene expression and signaling pathways.

\section{Introduction}

Crohn's disease $(\mathrm{CD})$ is a chronic, nonspecific inflammatory bowel disease (IBD) with unknown etiology and it can affect any part of the gastrointestinal tract from mouth to anus but mainly develops in the colon and the terminal ileum. Its main clinical manifestations are abdominal pain, diarrhea, and bloody purulent stool. The pathogenesis of
$\mathrm{CD}$ is not completely understood. Most studies suggest that the occurrence of $\mathrm{CD}$ is associated with genetics, immunity, environment, and intestinal microecology. As one type of cellular stress response, autophagy plays a critical role in innate immunity and adaptive immunity and is an important component of immune homeostasis. The autophagy pathway and biosynthesis together maintain the dynamic equilibrium of intracellular macromolecules [1] that play 
important roles in the development and progression of diseases. In recent years, the function of autophagy and some autophagy-associated genes in the development of IBD has been confirmed. Autophagy plays multiple roles in the pathogenesis of IBD by altering intracellular bactericidal effects, goblet cell function, proinflammatory cytokine production by macrophages, antigen presentation of dendritic cell, and endoplasmic reticulum stress response in intestinal cells [2].

Our previous studies indicated that herb-partitioned moxibustion (HPM) had good effect on the treatment of CD [3-6]; however, the mechanism of its effect requires further exploration. Therefore, the investigation of the pathogenic mechanism of CD and the immune-regulatory mechanism of HPM on CD with a focus on autophagy will be important in the study of pathogenesis, diagnosis, and treatment of $\mathrm{CD}$. The rapid development of transcriptomics, proteomics, and metabolomics provides new methods for investigating the mechanisms of acupuncture and moxibustion. The transcriptome is the sum of all RNAs transcribed in a developmental stage or functional status of specific tissues or cells. Transcriptomics can be used to study gene expression, structure, and function at a global level to reveal the molecular mechanism of specific biological or pathological processes. As a next-generation transcriptome sequencing technology, RNA sequencing (RNA-Seq) has the advantages of high throughput, high sensitivity, high resolution, and not being subject to sample restrictions, which can provide reliable technical support for studying gene structure and function. Therefore, our study used RNA-Seq to observe changes of autophagy- and immune-associated gene expression profiles in colon tissues of CD rats and the regulatory effect of HPM. It will provide new ideas for understanding the development of CD and the mechanism of HPM treatment from the perspective of autophagy.

\section{Materials and Methods}

2.1. Experimental Animals. Adult male Sprague-Dawley (SD) rats, weighing $180 \pm 20 \mathrm{~g}$, were provided by the Experimental Animal Center of Shanghai University of Traditional Chinese Medicine and were purchased from Vital River Laboratory Animal Technology Co., Ltd. (Beijing, China). The license for the use of experimental animals was SCXK (Beijing) 20120001 . All the animals were housed in a clean grade room with controlled temperature $\left(20 \pm 2^{\circ} \mathrm{C}\right)$, a light/dark $(12 \mathrm{~h}$ : $12 \mathrm{~h}$ ) cycle, and 50-70\% indoor humidity. All experimental protocols were approved by the Animal Research Ethics Committee of Shanghai University of Traditional Chinese Medicine.

2.2. Establishment of CD Model. After one week of adaptive feeding, the 60 rats were randomly divided into normal $(\mathrm{N})$, CD model (M), CD model with herb-partitioned moxibustion (MM), normal with herb-partitioned moxibustion (NM), CD model with mesalazine (western medicine, Med) group, and CD model with normal saline (NS) groups, with 10 rats in each group. The $\mathrm{CD}$ models were established by 2,4,6-trinitro-benzene-sulfonic acid (TNBS, Sigma, USA) according to Morris' method [7] except for the N and NM groups. The rats were fasted and given water for $24 \mathrm{~h}$, then rats were weighed and $2 \%$ sodium pentobarbital $(30 \mathrm{mg} / \mathrm{kg})$ was used for anesthesia through intraperitoneal injection. The rats were anally injected with 5\% TNBS solution mixed in $50 \%$ alcohol at a $1: 2$ ratio $(3 \mathrm{ml} / \mathrm{kg})$ using a rubber tube, with a deep of $6-8 \mathrm{~cm}$, and the head of the rat was pushed down for about $1 \mathrm{~min}$ to prevent loss of the injected solution. The injection was repeated every 7 days for 4 weeks. After the CD rat models were established, HE staining was used to observe whether the CD model was successfully established.

2.3. Moxibustion Intervention. After the experimental CD rat models were successfully established, the rats were exposed to different treatments. In the MM and NM groups, the Tianshu (bilateral, ST25) and Qihai (CV6) acupoints were selected [810]. The herbal cake was Chinese medicine powder (Coptis chinensis, Radix aconiti lateralis, Cortex Cinnamomi, Radix aucklandiae, Flos carthami, Salvia miltiorrhiza, and Angelica sinensis) mixed and stirred with yellow wine to form a thick paste; the herbal cake was prepared with $1 \mathrm{~cm}$ in diameter and $0.45 \mathrm{~cm}$ in thickness using a mold. The moxa cone was made of about $90 \mathrm{mg}$ using a mold. HPM treatment was performed with the moxa cone placed on the top of the herbal cake at the ST25 and CV6 acupoints and ignited. Two moxa cones were used at each acupoint for each treatment once daily for 7 days. The rats in the Med group were fed with mesalazine (Losan Pharma GmbH, Germany), which was prepared at the proportion of adult and rat of 1:0.018 [11], once a day for 7 days. The rats in the NS group were fed with normal saline, 2 $\mathrm{ml}$ per time and once a day for 7 days. The rats in the $\mathrm{N}$ and $\mathrm{M}$ groups did not receive any treatment but were grabbed and immobilized using the same method applied to other groups.

2.4. Sample Collection. Rats were anesthetized by intraperitoneal injection of $1 \%$ pentobarbital sodium $(30 \mathrm{mg} / \mathrm{kg})$. After anesthetization, the abdominal cavity was opened and 4-6 $\mathrm{cm}$ of distal colon was collected $1 \mathrm{~cm}$ from the anus. The colon was divided into three parts, one part was fixed in $10 \%$ neutral formalin fixative solution, and the other two parts were placed in cryotubes after cutting into pieces, then frozen in liquid nitrogen for $1 \mathrm{~h}$, and later stored in a $-80^{\circ} \mathrm{C}$ freezer.

2.5. Histological Observation and Microscopic Scoring. Rat colon tissues were fixed in $10 \%$ neutral formalin fixative solution for $24 \mathrm{~h}$, dehydrated, embedded in paraffin, sectioned at a thickness of $4 \mu \mathrm{m}$, and subjected to HE staining. Histological changes of the colon tissues were observed under an optical microscope (BX33, Olympus) and scoring of pathological injury was performed according to the Score Criteria of Colonic Histological Damage [12].

2.6. RNA Extraction and Quality Control. Two colon tissue samples were randomly selected from each group, and total RNA was extracted using the Trizol (Bio-Rad, USA) method. The purity, concentration, and integrity of the total RNA were determined. Quality control of the total RNA was performed using the Agilent 2100 Bioanalyzer. Samples with an RNA integrity number higher than 8 and a concentration $\geq 500 \mathrm{ng} / \mu \mathrm{L}$ were used for library construction and RNA-Seq. 
2.7. Construction of the RNA Library. A certain amount of the total RNA was collected, and the DNA fragments present in the total RNA sample were digested using DNase I. mRNA was purified using oligo (dT) magnetic beads (Invitrogen, USA) and then eluted using a buffer solution and placed on ice. A breaking agent was added, and the mixture reacted in a Thermomixer at an appropriate temperature for the specified time. The sample was precipitated, and the broken product was recovered. The broken mRNA was mixed thoroughly with an appropriate amount of primers, and the mixture was placed in a Thermomixer at an appropriate temperature for a certain amount of time to allow for secondary structure opening for primer binding. The preprepared first-chain synthesis reaction sample was added, and the first-chain cDNA was synthesized in a PCR machine using the corresponding program. Second-chain cDNA was also synthesized using the same method. After end repair, the sample was dissolved in EB solution. Next, an adenine was added to the cDNA 3' end, and an adaptor was ligated to the cDNA 5' end. The ligated product was subject to agarose gel electrophoresis. The gel was cut according to the size of the DNA fragment. The PCR sample was prepared, and an appropriate PCR program was selected to amplify the obtained ligated product to complete the construction of RNA library.

2.8. RNA Sequencing and Data Analysis. The quality of the RNA library was detected using the Agilent 2100 and ABI Step One Plus Real-Time PCR System, then the samples were processed and sequenced using the Hiseq400 sequencing platform. Data collection software provided by Illumina was used to control the sequencing process and perform realtime data analysis. For the comparison between two groups, differentially expressed genes were defined as genes with a fold change $(F C) \geq 1.5$. The $\log _{2}$ ratio value was the normalized $\log _{2}$ FC value, i.e., $\mid \log _{2}$ ratio $\mid \geq 0.58$. For example, a $\log _{2}$ ratio value of group $\mathrm{A} /$ group $\mathrm{B}$ higher than 0.58 indicated that the gene was upregulated in group A compared to that in the group $\mathrm{B}$ and $\log _{2}$ ratio value lower than -0.58 indicated that the gene was downregulated in group A compared to that in the group B.

2.9. Validation of Differentially Expressed Gene Using qRT$P C R$. Total RNA extraction was performed using Trizol method, and the selected differentially expressed genes were validated through reverse transcription and real-time PCR amplification. The $2^{-\Delta \Delta \mathrm{Ct}}$ method was performed to analyze relative mRNA expression in samples of colon tissue.

2.10. Statistical Analysis. The SPSS 18.0 software was used for the data analysis. The data were presented as the mean \pm standard deviation $(\bar{x} \pm s)$ and the comparison of differences among groups was performed using one-way ANOVA if data conformed to the normal distribution. If the variances were homogeneous, the pairwise comparison was performed using the least significant difference (LSD) test, and Dunnett's T3 method was performed if the variances were not homogenous. Data that did not conform to the normal distribution were presented as the median and quartiles $[\mathrm{M}(\mathrm{P} 25, \mathrm{P} 75)]$ and Nonparametric Kruskal Wallis test was performed for the comparison of differences among groups. The significance level of the statistical examination was $\alpha=0.05$. $P<0.05$ indicated that the difference had statistical significance.

\section{Results}

3.1. Changes of the Body Weights of Rats. The body weights of the rats had no significant differences among the groups before the model establishment. After the CD model was established, the body weights of the rats in the $M$ group, MM group, Med group, and NS group were significantly decreased compared with the $\mathrm{N}$ group $(P<0.01$ or $P<0.05)$, while there was no significant difference between the $\mathrm{N}$ group and the NM group rats; the body weights of the rats in the $\mathrm{M}$ group, MM group, and NS group were also significantly decreased compared with the NM group $(P<0.01$ or $P<0.05)$. After the treatment, the body weights of the rats in the $M$ group were still significantly decreased compared with the $\mathrm{N}$ group $(P<0.01)$; the body weights of the rats in the MM group, Med group, and NS group were significantly increased compared with the $\mathrm{M}$ group $(P<0.01)$; there was also no significant difference between the $\mathrm{N}$ group and the NM group rats (Supplementary Figure S1).

\subsection{Histological Changes and the Injury Scores of Colon}

Tissues. In the $\mathrm{N}$ and NM group, the epithelial structure of the colon tissues was clear, with intact and continuous colon mucosa, the arrangement of glands was ordered, a small number of inflammatory cells were observed, and there was no hyperemia, edema, hyperplasia, or ulcer appeared. In the $M$ group, monocyte-macrophage hyperplasia was observed in the submucosal layer of the colon tissues, with dilation, hyperemia, edema of the surrounding blood vessel, disordered tissue structure, gland dilation, and granuloma formation. In the MM group, the mucosal epithelium of the colon tissues was more intact than the $\mathrm{M}$ group, some glands were lost with heal shallow ulcers, and there was a few inflammatory cells and fibrocytes infiltration. In the Med group, the colon mucosal epithelial was covered by hyperplasia tissues, formation of small healing ulcers, atypical hyperplasia of glands on the ulcer edge, a large number of fibroblasts in the submucosal layer, and hyperplasia of new capillaries. In the NS group, a large amount of mucosa was lost and presented as giant ulcers that reach the muscular layer, atypical hyperplasia of gland epithelial cells, cell exudation and necrosis on the surface of ulcers, and a large number of granulomas at the bottom of ulcers (Figure 1).

The pathological injury scores showed that the colon injury scores in the M group were significantly higher than those of the normal group, and the difference was statistically significant. While the colon injury scores in the MM and Med groups were both significantly decreased compared to the $M$ group (Figure 2).

\subsection{Correlation Analyses of Gene Expression between Samples.} The Euclidean distance calculation method was used to calculate the distance of gene expression levels in all samples. As shown in Figure 3, the vertical axis indicates the height of the clustering tree. Samples with similar heights were 


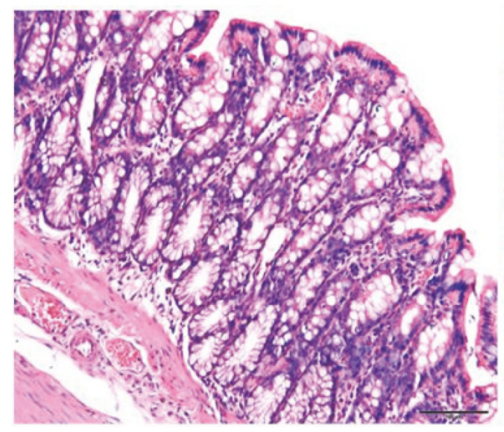

(a)

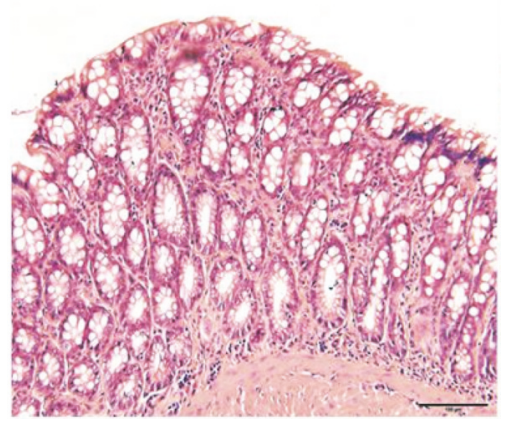

(d)

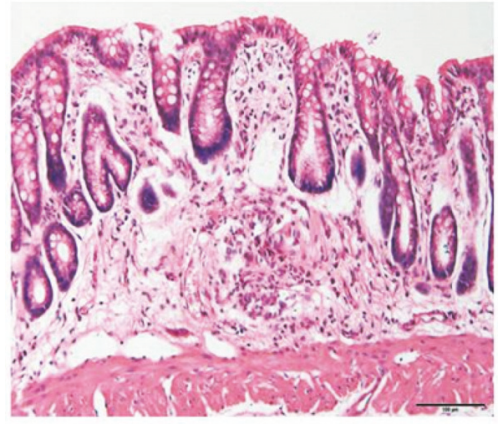

(b)

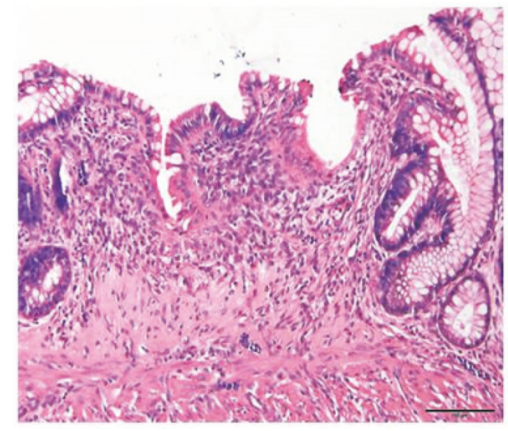

(e)

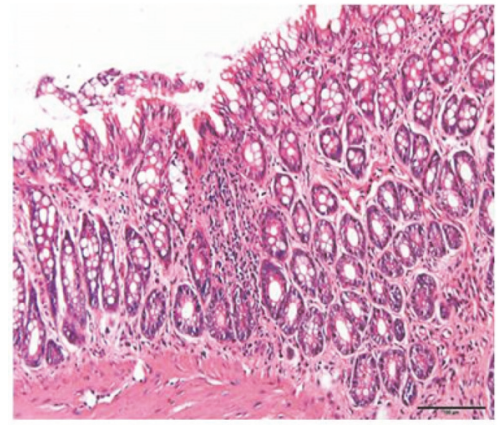

(c)

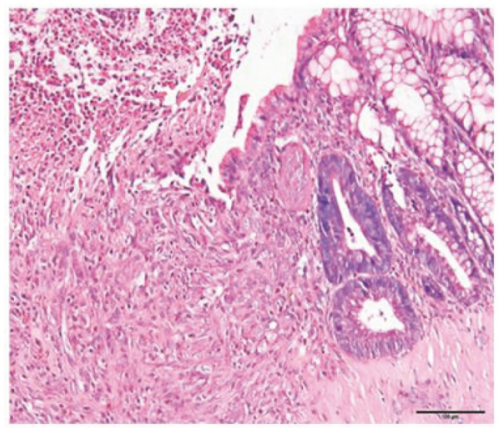

(f)

FIGURE 1: The histological observation of rat colon in each group by HE staining method, $(200 \times)$. (a) Normal group; (b) CD model group; (c) CD model with herb-partitioned moxibustion group; (d) Normal with herb-partitioned moxibustion group; (e) CD model with mesalazine group; (f) CD model with normal saline group.

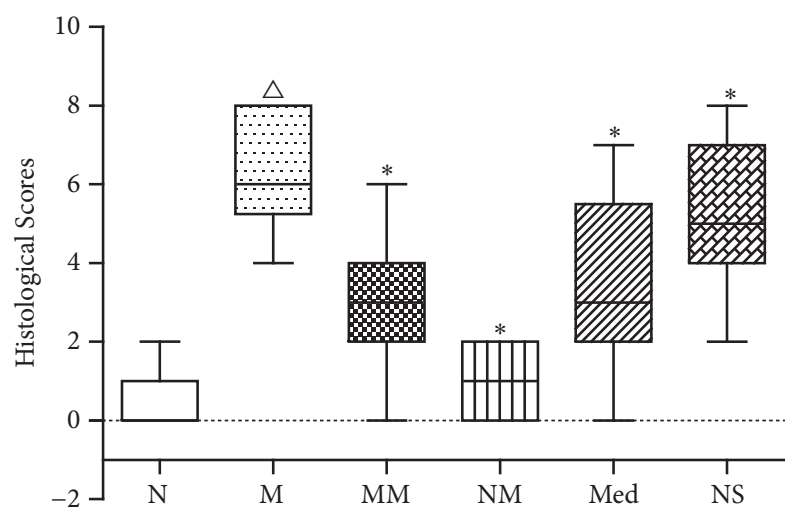

Figure 2: Histological scores of rat colon in each group. N: normal group; M: CD model group; MM: CD model with herbpartitioned moxibustion group; NM: normal with herb-partitioned moxibustion group; Med: CD model with mesalazine group; NS: CD model with normal saline group. ${ }^{\triangle} P<0.01$ versus normal group; ${ }^{*} P<0.01$ versus model group. $\mathrm{P}$ values between different groups were calculated from nonparametric test.

prone to cluster together. The distance between samples was also calculated using the square sum of deviations method (Ward's method). A cluster plot was established to represent the distances between samples, which intuitively reflected the difference between samples. Our result shows that the CD model group and all the treatment groups generally belonged to two classes. The difference between the CD model group and the $\mathrm{N}$ group was significant, while the difference among the MM group, NM group, and the N group was smaller.

In addition, we performed heatmap analyses on the correlation between samples (Supplementary Figure S2). When the correlation value was smaller (closer to 0 ), the color was close to white; when the correlation value was larger (closer to 1), the color was close to blue. It showed that the difference between NM1 and Med2 was largest. Overall, the differences between the $\mathrm{N}$ group and the $\mathrm{M}$ group as well as the NM group and the M group were larger, while the differences between the Med group and the M group as well as the NS group and the M group were smaller.

\subsection{Analysis of Significantly Differential Genes between} Groups. Based on the whole gene expression of the RNA-Seq, the comparison of gene expression between different groups was observed. The comparison between the normal group and the CD model group showed 42 upregulated genes and 39 downregulated genes. The comparison between the MM group and the CD model group showed 93 upregulated genes and 93 downregulated genes. The comparison between the NM group and CD model group showed 126 upregulated genes and 158 downregulated genes. The comparison between the Med group and CD model group showed 57 upregulated genes and 83 downregulated genes. The comparison between the NS group and CD model group showed 80 upregulated genes and 41 downregulated genes. The comparison between 


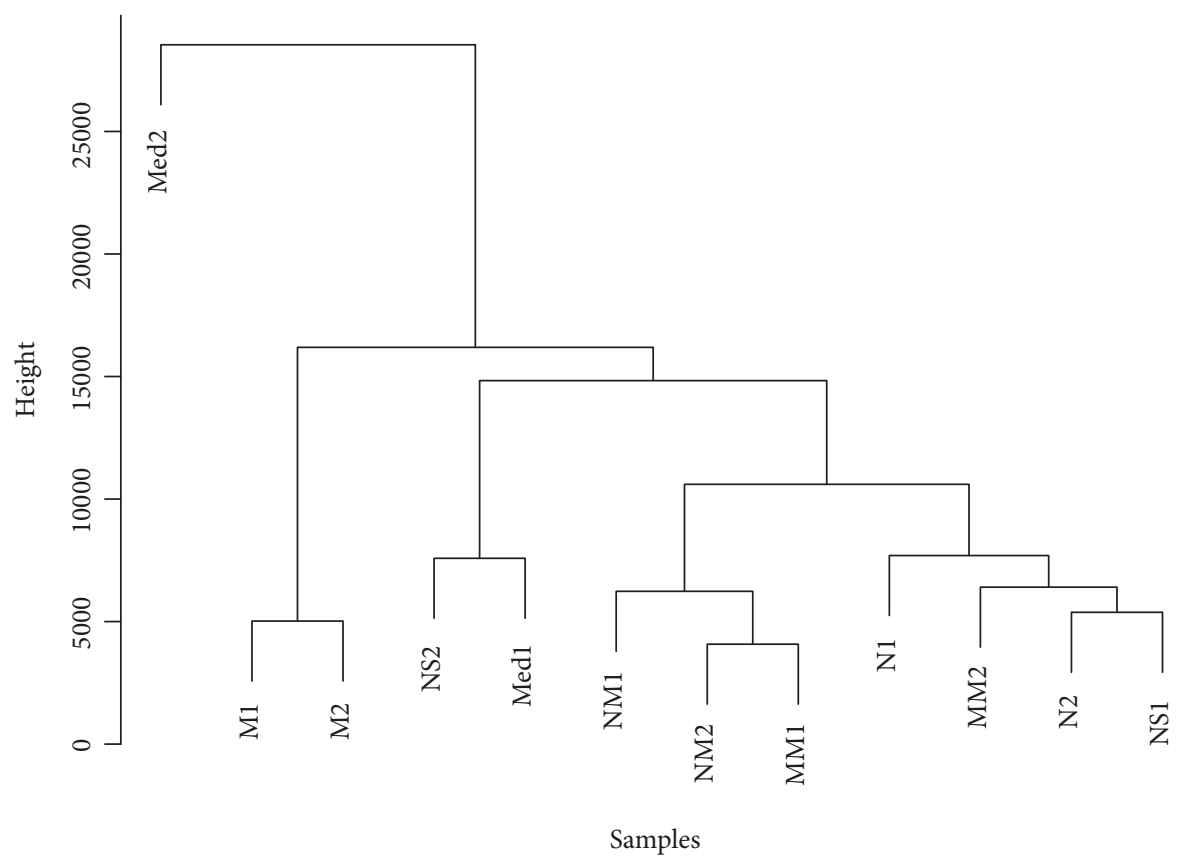

FiguRE 3: Clustering tree of distances between different groups. N: normal group; M: CD model group; MM: CD model with herb-partitioned moxibustion group; NM: normal with herb-partitioned moxibustion group; Med: CD model with mesalazine group; NS: CD model with normal saline group.

the MM group and Med group showed 110 upregulated genes and 86 downregulated genes (Supplementary Figure S3).

Next, cluster analyses were performed on significantly differentially expressed genes (Figure 4). The differential genes in the MM group, the NM group, and the Med group had low levels of upregulation or downregulation compared to the $\mathrm{N}$ group, indicating similar gene expression between each of these groups and the $\mathrm{N}$ group. While the upregulation of genes in the NS group was significant compared to that in the normal group, the differences of gene expression between the NS group and NC group were larger. The differential genes in the MM group had high levels of upregulation or downregulation with respect to the $\mathrm{M}$ group, indicating that there were large differences of gene expression between the MM group and the M group. Compared to the M group, the overall colors of differential genes were relatively lighter in the Med group and the NS group, suggesting that the levels of upregulation and downregulation of differential genes in the Med group and the NS group were lower with respect to the $\mathrm{M}$ group.

3.5. Analysis of Differential Gene Expression Profiles of Autophagy- and Immune-Associated Molecules between Groups. We further calculated the gene expression level of each sample and the expression levels of detected genes between different groups were compared. The differentially expressed genes were defined as those with a difference $\geq 1.5$-fold change. Compared to the $\mathrm{N}$ group, the $\mathrm{M}$ group had 11 genes that were upregulated and two genes that were downregulated in which the $\mathrm{CD}$ autophagy-associated gene
Nod 2 was upregulated 1.357-fold, Irgm was upregulated 1.106fold, and Atg $9 b$ was upregulated 2.459-fold; the immuneassociated cytokine gene Il12b was upregulated 6.119-fold, Il22 was upregulated 3.858-fold, and Il23r was downregulated 1.322 -fold (Table 1). Compared to M group, the MM group had five genes that were upregulated and eight genes that were downregulated in which $\mathrm{Nod} 2$ was downregulated 0.902 -fold, Irgm2 was downregulated 0.68 -fold, and Atg9b was downregulated 2.459-fold; the immune-associated cytokine gene Il22ra2 was downregulated 2.324-fold, Il22ra1 was downregulated 0.613 -fold, and Il12rb1 was downregulated 1.067-fold (Table 2). Compared to the M group, the Med group had four genes that were upregulated and 15 genes that were downregulated in which Nod 2 was downregulated 0.771-fold, Irgm was downregulated 0.84-fold, the immuneassociated cytokine gene Il22 was downregulated 3.858-fold, and Il12b was downregulated 3.119-fold, while $T g f b 1$ and $\mathrm{Tgfb2}$ of, the TGF family, were downregulated 1.548-fold and 0.996-fold, respectively (Table 3). Compared to the M group, the NS group had eight genes that were upregulated and 10 genes that were downregulated, in which Atg $9 b$ was downregulated 0.874 -fold, the immune-associated cytokine gene Ifng was upregulated 5.426-fold, Il27ra was upregulated 2.015 -fold, and Il21 was upregulated 1.216-fold (Table 4).

3.6. Pathway Enrichment of Differential Genes. The pathway enrichment results for differential genes (Figure 5) showed that the pathways with higher enrichment levels between the $\mathrm{N}$ group and the $\mathrm{M}$ group included the cell adhesion molecule (CAM) pathway and the Ribosome pathway. The pathways with higher enrichment levels between the MM group and the 
TABLE 1: Differential expression of autophagy- and immune-associated genes between N group and M group. N: normal group, M: CD model group.

\begin{tabular}{|c|c|c|c|}
\hline \multicolumn{2}{|c|}{ Upregulation } & \multicolumn{2}{|c|}{ Downregulation } \\
\hline Gene name & $\begin{array}{c}\log 2 \text { Ratio } \\
(\mathrm{M} / \mathrm{N})\end{array}$ & Gene name & $\begin{array}{c}\log 2 \text { Ratio } \\
(\mathrm{M} / \mathrm{N})\end{array}$ \\
\hline $\mathrm{Il} 12 \mathrm{~b}$ & 6.119 & Il23r & -1.322 \\
\hline $\mathrm{I} 122$ & 3.858 & LOC685590 & -0.926 \\
\hline Atg9b & 2.459 & & \\
\hline Htr2b & 2.225 & & \\
\hline Il21r & 2.129 & & \\
\hline LOC103690381 & 1.931 & & \\
\hline Nod2 & 1.357 & & \\
\hline Tsc22d 4 & 1.219 & & \\
\hline Slc44a2 & 1.173 & & \\
\hline Irgm & 1.106 & & \\
\hline Slc25a4 & 0.839 & & \\
\hline
\end{tabular}

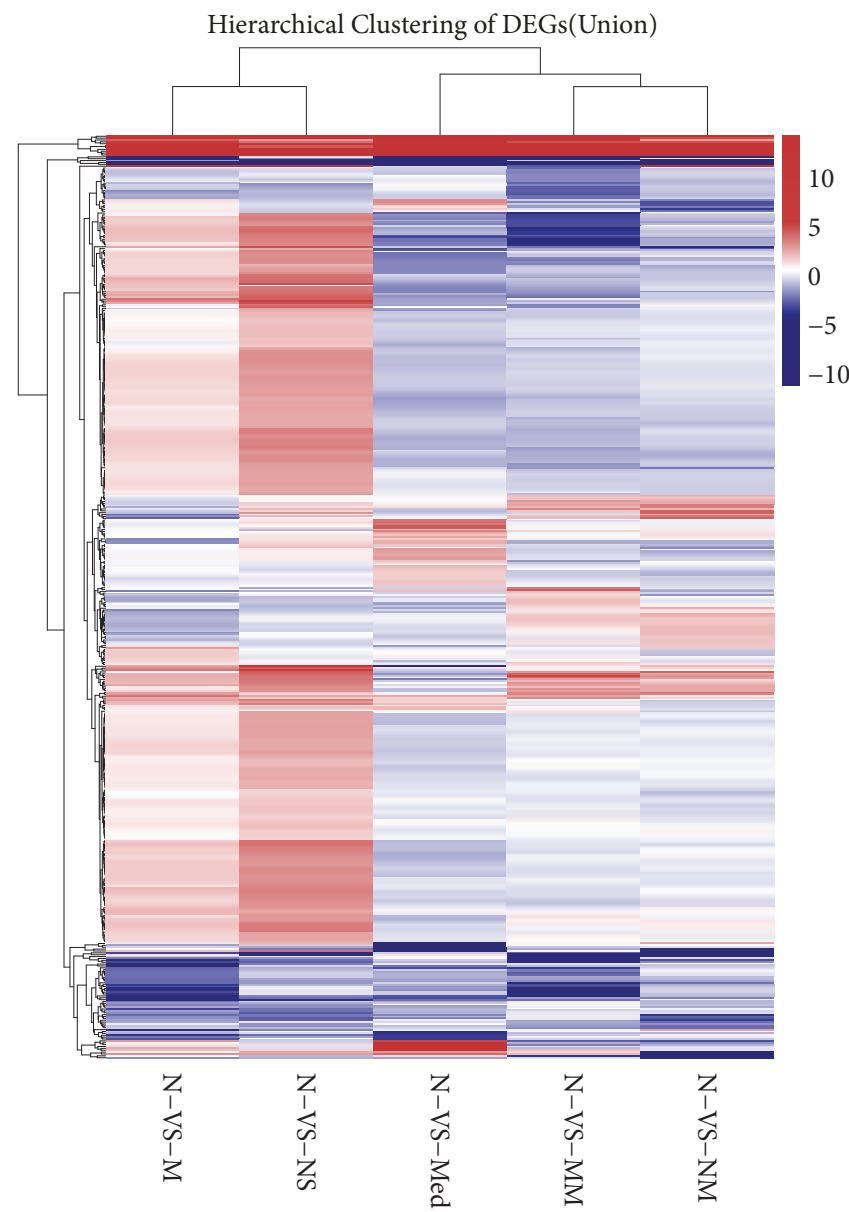

(a)

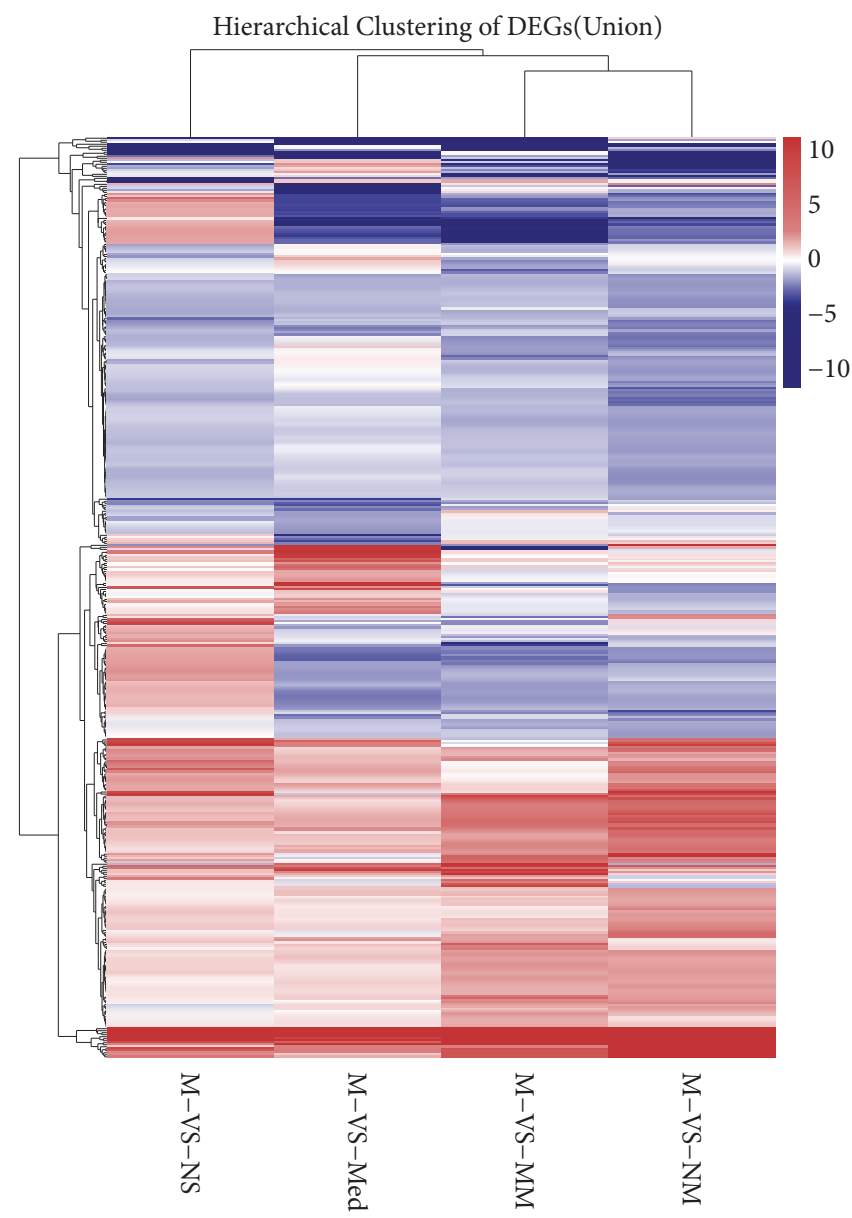

(b)

Figure 4: Heatmap of the clustering expression profiles of differential genes in all groups. (a) Comparison of differential gene expression between normal group and other groups. (b) Comparison of differential gene expression between CD model group and other groups. The gradient map indicates the differential fold values after logarithmic conversion. Each column indicates one differential pair, and each row indicates one differential gene. Different types of differential genes are represented by different colors: red indicates upregulation and blue indicates downregulation. The levels of upregulation and downregulation increased with the increasing darkness of the color. N: normal group; M: CD model group; MM: CD model with herb-partitioned moxibustion group; NM: normal with herb-partitioned moxibustion group; Med: CD model with mesalazine group; NS: CD model with normal saline group. 
TABLE 2: Differential expression of autophagy- and immune-associated genes between MM group and M group. MM: CD model with herbpartitioned moxibustion group, M: CD model group.

\begin{tabular}{lccc}
\hline Upregulation & log2 Ratio & & $\begin{array}{c}\text { Downregulation } \\
\text { Gene name }\end{array}$ \\
(MM/M) & Gene name & -5.714 \\
Rn5s & 4.247 & Il21 & -3.368 \\
Mmp10 & 4.076 & Il21r & -2.459 \\
LOC100363136 & 3.930 & Atg9b & -2.324 \\
Tff3 & 3.807 & Il22ra2 & -1.067 \\
Zg16 & 3.705 & Il12rb1 & -0.902 \\
& & Nod2 & -0.837 \\
& & LOC685590 & -0.68 \\
& & Irgm2 & -0.659 \\
& & Atg14 & -0.62 \\
& & Ifnlr1 & -0.613 \\
& & Il22ral & -0.582 \\
\hline
\end{tabular}

TABle 3: Differential expression of autophagy- and immune-associated genes between Med group and M group. Med: CD model with mesalazine group; M: CD model group.

\begin{tabular}{|c|c|c|c|}
\hline \multicolumn{2}{|c|}{ Upregulation } & \multicolumn{2}{|c|}{ Downregulation } \\
\hline Gene name & $\begin{array}{c}\log 2 \text { Ratio } \\
(\mathrm{Med} / \mathrm{M})\end{array}$ & Gene name & $\begin{array}{c}\log 2 \text { Ratio } \\
(\mathrm{Med} / \mathrm{M})\end{array}$ \\
\hline Ill7a & 2.907 & $\mathrm{Il} 22$ & -3.858 \\
\hline Ill7b & 1.285 & $\mathrm{Il} 12 \mathrm{~b}$ & -3.119 \\
\hline Il $23 r$ & 1.280 & Tnfrsf18 & -2.157 \\
\hline Ill7rd & 0.606 & Tnfsf9 & -1.776 \\
\hline & & Tgfb1 & -1.548 \\
\hline & & Tgfb2 & -0.996 \\
\hline & & Ill7d & -0.945 \\
\hline & & Il12rb1 & -0.884 \\
\hline & & Tgfb3 & -0.866 \\
\hline & & Irgm & -0.84 \\
\hline & & Il22ra2 & -0.8 \\
\hline & & Nod2 & -0.771 \\
\hline & & Tgfbr2 & -0.717 \\
\hline & & Ifnarl & -0.706 \\
\hline & & Tgif1 & -0.617 \\
\hline
\end{tabular}

TABLE 4: Differential expression of autophagy- and immune-associated genes between NS group and M group. NS: CD model with normal saline group; M: CD model group.

\begin{tabular}{lcr}
\hline Upregulation & $\begin{array}{c}\text { log2 Ratio } \\
(\mathrm{NS} / \mathrm{M})\end{array}$ & $\begin{array}{c}\text { Downregulation } \\
\text { Gene name }\end{array}$ \\
\hline Ifng & 5.426 & Atg9b \\
Il27ra & 2.015 & -0.874 \\
LOC685590 & 1.895 & \\
Il21r & 1.653 & \\
Il21 & 1.216 & \\
Il23r & 1.147 & \\
Ill7b & 0.736 & \\
Tgfbr1 & 0.620 & \\
\hline
\end{tabular}




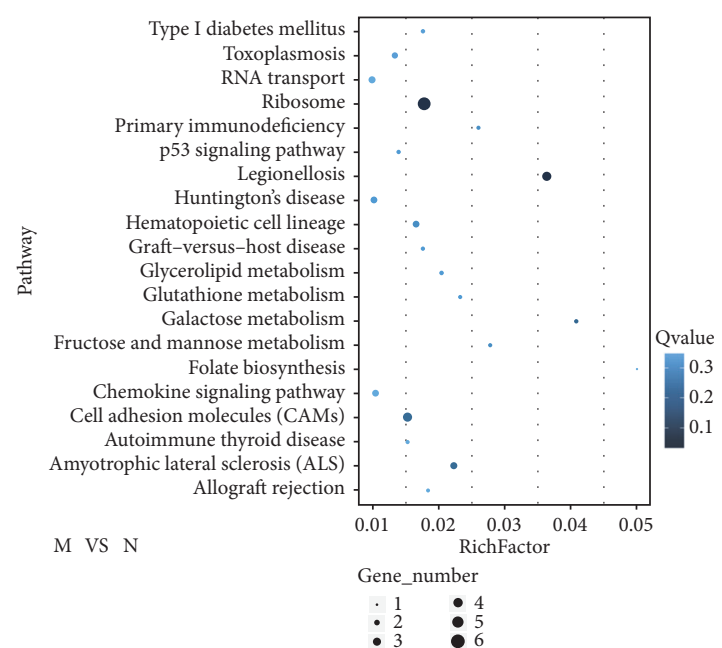

(a)

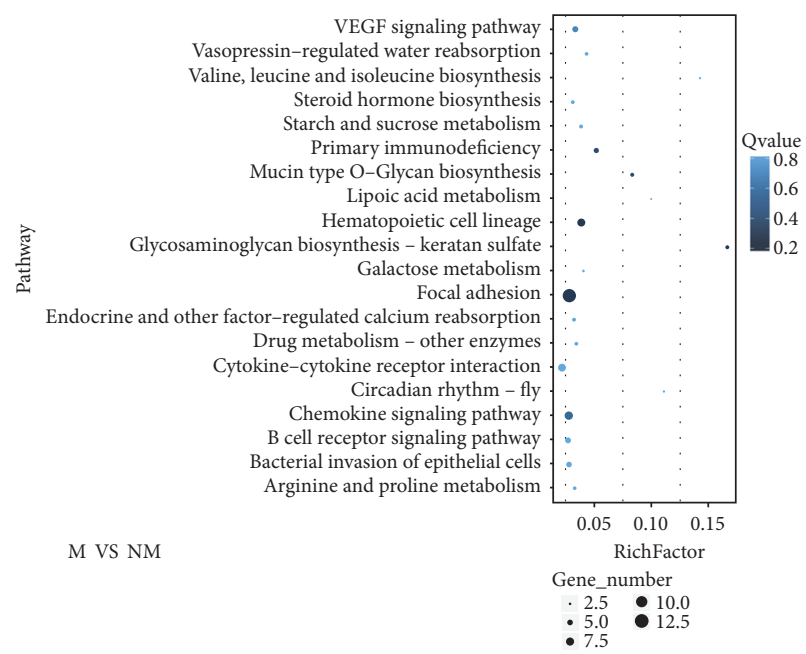

(c)

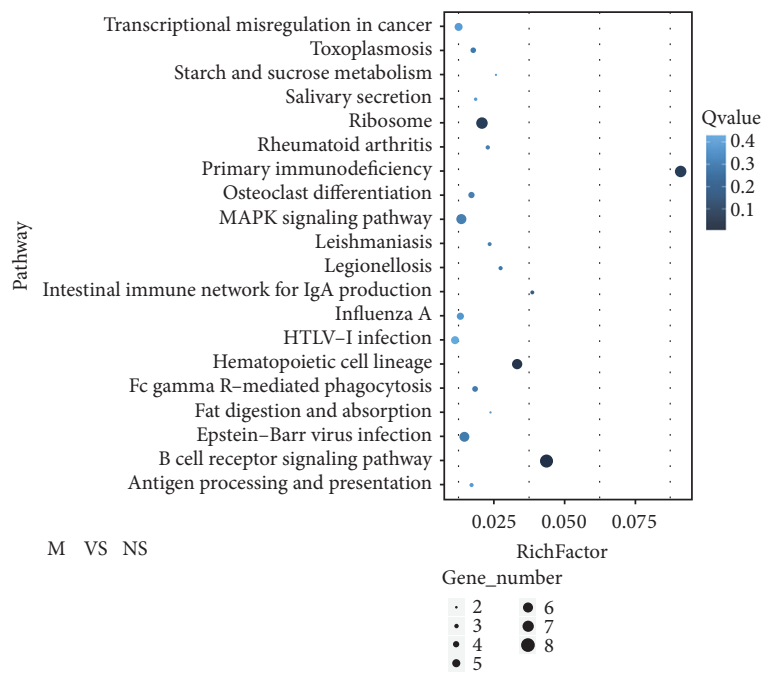

(e)

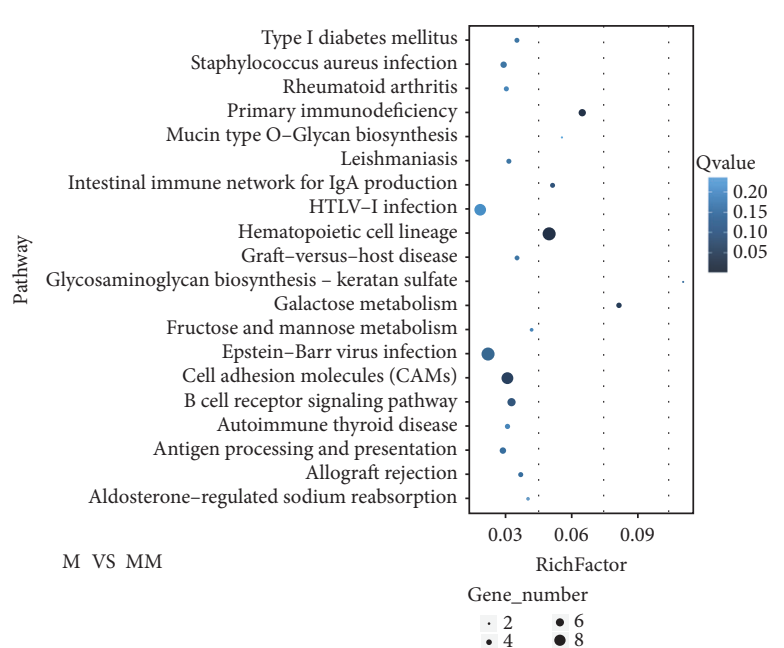

(b)

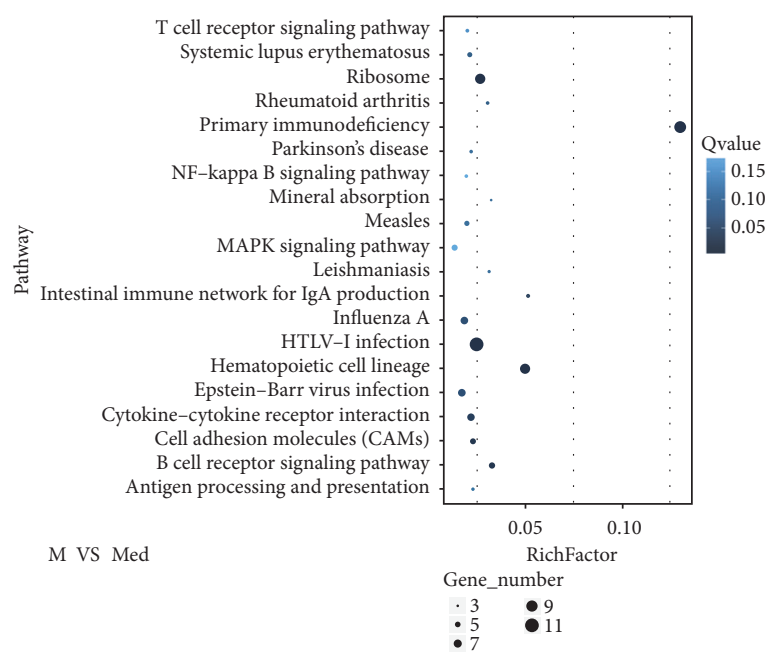

(d)

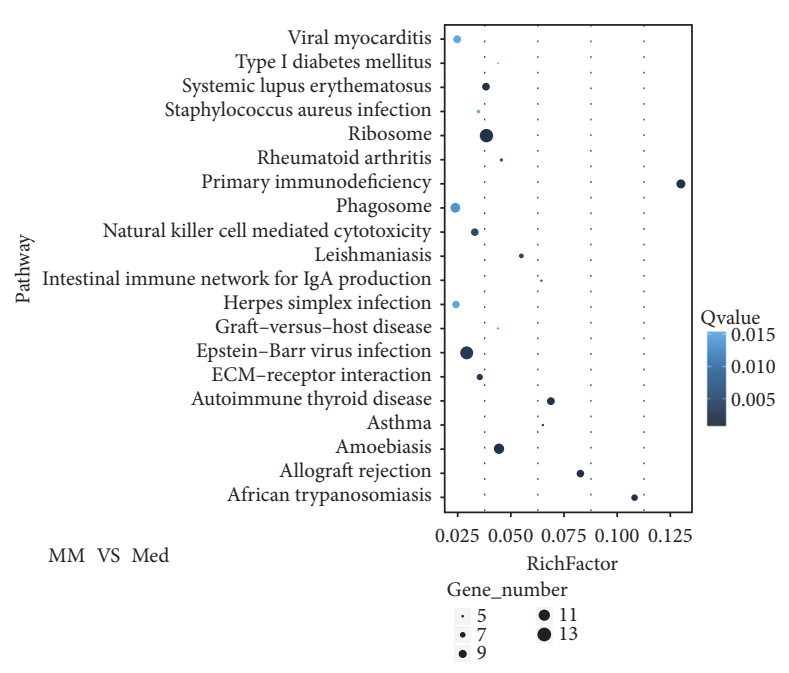

(f)

FIGURE 5: The scatter plot of pathway enrichments of differential genes between groups. (a) M group versus N group. (b) M group versus MM group. (c) M group versus NM group. (d) M group versus Med group. (e) M group versus NS group. (f) MM group versus Med group. The enrichment factor refers to the ratio between the number of differentially expressed genes in the pathway item and the total number of annotated genes in the same pathway item. When the enrichment factor was larger, the enrichment level was higher. The $Q$ value was the normalized $\mathrm{P}$ value after multiple hypothesis testing, and the range of values was 0 to 1 . When the value was close to 0 , the enrichment was more significant. The figure only shows the pathway items with the top 20 enrichment levels. N: normal group; M: CD model group; MM: CD model with herb-partitioned moxibustion group; NM: normal with herb-partitioned moxibustion group; Med: CD model with mesalazine group; NS: CD model with normal saline group. 


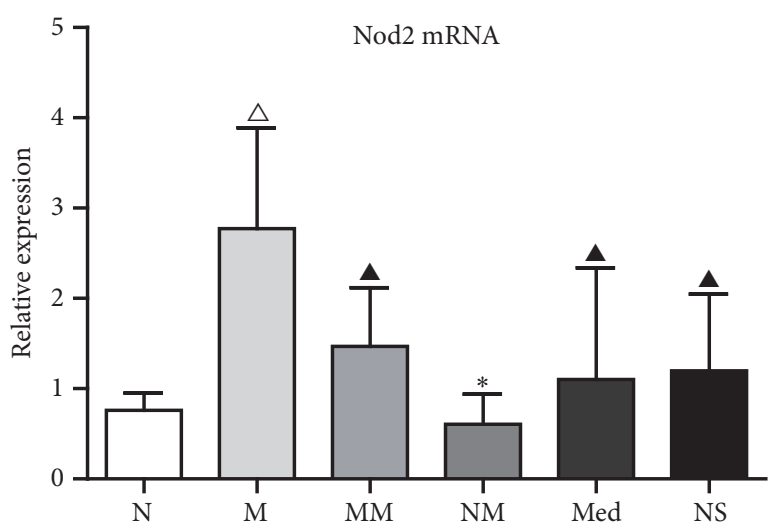

(a)

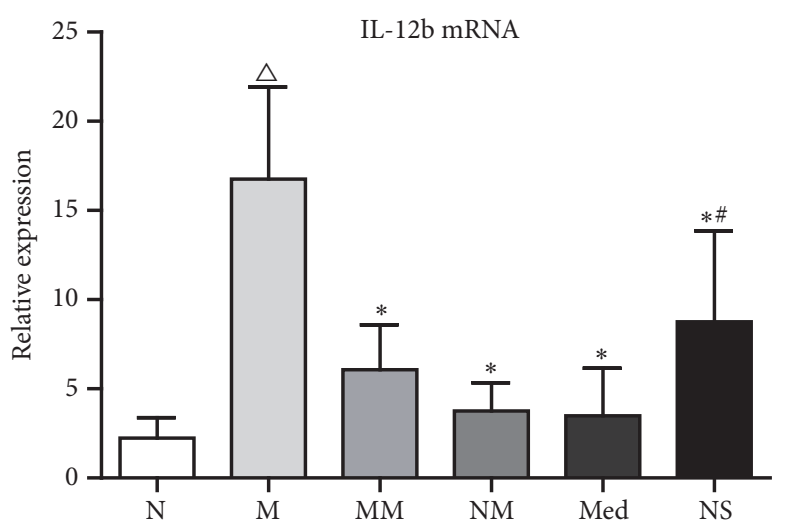

(c)

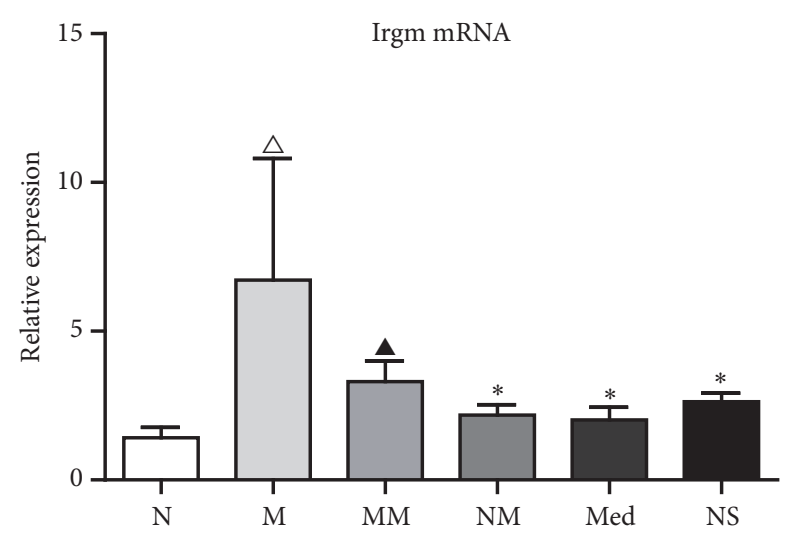

(b)

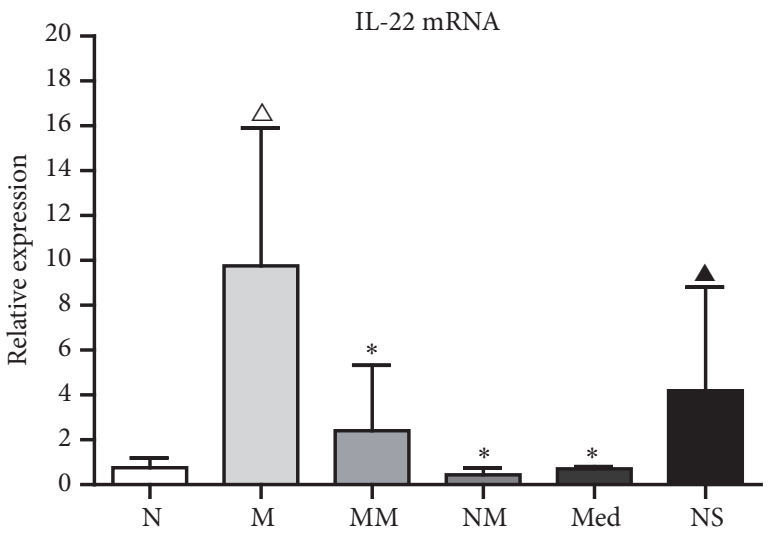

(d)

Figure 6: The expression of differential expression mRNA in colon tissues of rats. (a) The expression of Nod2 mRNA in rat colon tissues. (b) The expression of Irgm mRNA in rat colon tissues. (c) The expression of IL-12bmRNA in rat colon tissues. (d) The expression of IL-22 mRNA in rat colon tissues. N: normal group; M: CD model group; MM: CD model with herb-partitioned moxibustion group; NM: normal with herb-partitioned moxibustion group; Med: CD model with mesalazine group; NS: CD model with normal saline group. ${ }^{\triangle} P<0.01$ versus N group; ${ }^{\wedge} P<0.05$ versus M group; ${ }^{*} P<0.01$ versus M group; ${ }^{*} P<0.05$ versus Med group. $P$ values between different groups were calculated from one-way ANOVA and LSD test.

model group included HTLV-1 infection pathway, EpsteinBarr virus infection, and CAM pathway. While the pathways with higher enrichment levels between the Med group and the model group included MAPK signaling pathway, B cell receptor signaling pathway, the pathways with higher enrichment levels between the NS group and the model group included MAPK signaling pathway, HTLV-1 infection pathway, Ribosome pathway, the pathways with higher enrichment levels between the MM group and the Med group included Ribosome pathway, Phagosome pathway.

3.7. Validation of Differential Expression mRNA in Colon Tissues of Rats. According to the results of analysis of differential gene expression profiles, we found that the expression of Nod2, Irgm, Il-12b, and Il-22 genes in the M group increased compared to the N group, and HPM and mesalazine both downregulated the expression of autophagy-associated Nod2, Irgm. Interestingly, mesalazine treatment downregulated the expression of Il-12b, Il-22 genes while HPM treatment downregulated the expression of $\mathrm{Il}-12 \mathrm{~b}, \mathrm{Il}-22$ receptor genes of $\mathrm{Il}-$ $12 \mathrm{rb} 1$ and Il-22ra2. Finally we selected the Nod2, Irgm, IL$12 \mathrm{~b}, \mathrm{IL}-22$ for validation using the qRT-PCR. The expression levels of Nod2, Irgm, IL-12b, and IL-22 mRNA were increased in the $\mathrm{M}$ group compared to the $\mathrm{N}$ group $(P<0.01)$. While the expression of Nod2, Irgm, IL-12b, IL-22 mRNA were decreased in the MM and Med groups compared to the $\mathrm{M}$ group $(P<0.01$ or $P<0.05)$ (Figure 6).

\section{Discussion}

$\mathrm{CD}$ is a chronic, intractable, intestinal disease. Currently, the western medicine treatments of $\mathrm{CD}$ mainly include salicylic acid preparations, hormones, immunosuppressive agents, biological agents, antibacterial agents, and probiotics. However, long-term use of western medicine will produce obvious side effects, and recurrence is frequently observed after drug withdrawal. Therefore, we need treatment methods 
that are effective and convenient, have limited side effects, and are accepted by patients. Previous studies showed that acupuncture and moxibustion have definite efficacy on CD; especially that HPM could attenuate abdominal pain and diarrhea in mild to moderate CD patients [13-15]. In this study, HPM significantly improved the pathological injury of colon tissues in CD rats, which was consistent with previous studies $[5,16]$.

The pathogenic mechanism of CD is a complex process and genetic factors play an important role in the development of CD [17]. Genome-wide association studies (GWAS) and meta-analysis showed that there are 163 IBD-associated genomes [18] and 71 CD-associated genomes [19]. In 2001, Hugot et al. [20] and Ogura et al. [21] reported that the NOD2 gene (also known as CARD) at the OBD1 locus was the first CD-susceptibility gene in humans. Subsequently, single nucleotide polymorphisms of autophagy-associated genes of ATG16L1 [22], IRGM [23], and ULK1 [24] were found to affect autophagy and were closely associated with the development of CD [25].

Therefore, we performed RNA-Seq to observe the changes of gene expression in colon tissues of $\mathrm{CD}$ rats and the regulating effect of HPM. The results showed that HPM and mesalazine treatments both reduced the high expression levels of autophagy-associated genes of Nod2, $\operatorname{Irgm}$, while mesalazine treatment also downregulated the Atg9b gene expression in colon of CD rats. The high expression levels of Nod2, Irgm mRNA in the CD model were also decreased by HPM and mesalazine treatments. NOD2 belongs to the nucleotide-binding oligomerization domain-like receptor protein family and is an intracellular pattern recognition receptor of the Nod-like receptor family [26]. ATG16L1 is a risk allele gene of $\mathrm{CD}$; its single nucleotide polymorphisms (SNPs) associated with autophagy are the key factor in the autophagic response to invading pathogens and increasing the risk of CD [27]. Autophagy mediated by ATG16L1 plays an important role in the intestinal epithelium in CD. Intestinal epithelial Paneth cells exhibit notable abnormalities in the granule exocytosis pathway in ATG16L1-deficient mice, and the functional changes of many biological processes that these cells involved in directly influence intestinal injury [28]. While NOD2 can mediate autophagy and the pathological process of CD through ATG16L1, mainly in ATG16L1 can inhibit the production of inflammatory cytokines by NOD2 via its autophagy [29]. ATG9b is also an autophagy gene. The phagophores driven by ATG9b can promote the binding of LC3 and p62 to initiate autophagy-associated degradation. ATG9b deficiency can block the recruitment of p62associated ubiquitinated protein for autophagic lysosome degradation to participate in autophagy regulation [30]. IRGM is both an autophagy gene and a risk gene of IBD. The rs13361189 and rs4958847 gene polymorphisms within IRGM both have important effects on the development of $\mathrm{CD}$ and its features of clinical phenotypes [31]. Studies found that IRGM activates core autophagy, and it can couple to innate immune receptors. Microbial products or bacterial invasion can result in increased IRGM expression and AMPK stability. Specific protein-protein interactions and the ubiquitination of IRGM can activate the interactions of IRGM and the key autophagy regulator ULK1 and the human tumor suppressor gene Beclin1 (BECN1) and promote their coassembly, thus governing the formation of autophagy initiation complexes. Furthermore, IRGM can interact with ATG16L1 and NOD2 of CD risk factors to form a molecular complex. The interaction between IRGM, NOD2 and other pattern recognition receptors such as NOD1, RIG-I, and certain TLRs can transduce microbial signals to the core autophagy apparatus to control autophagy development $[32,33]$.

Immune factors are also important in the pathogenic mechanism of CD [34-36]. Invasion of various pathogens and harmful microbes destroys the intestinal mucosal epithelial barrier to increase the intestinal mucosal permeability, induce overreaction of intestinal immune system, release a series of cytokines and inflammatory mediators, activate immune response, and result in the CD tissue injury, thus inducing a series of pathological changes and clinical manifestations. Therefore, in addition to autophagy-associated genes, we focused on the expression of immune-associated cytokine genes. The results showed that the expression of $I l-12 b, I l-22$ genes in the colon tissues of CD rats were significantly increased, and mesalazine and HPM treatment respectively reduced the expression of $I l-12 b, I l-22$ genes and their receptor of $\mathrm{Il}-12 \mathrm{rb} 1$ and $\mathrm{Il}-22 \mathrm{ra} 2$ genes. In addition, we only selected the IL-12b and IL-22 for further validation based on the result of gene expression profile and the positive correlation between cytokines and their receptors [37]. Our results showed that the level of IL-12b and IL-22 mRNA were decreased through HPM and mesalazine treatment. IL-12 is an immune cell growth-stimulating factor in the interleukin12 family with many biological activities. IL- 12 can promote the differentiation and proliferation of $\mathrm{T}$ lymphocytes and NK cell, regulate cellular immunity, and increase the killing function of NK/LAK cells and the response ability of specific CTL cells [38]. IL-12b1 and IL-12b2 are the two subunits of IL-12 consisting of the functional IL12 receptor complex, and two subunits, IL-12rb1 and IL-12rb2 of the IL-12 receptor, can influence the response of $\mathrm{T}$ cells to IL-12. The IL12/IL-12R signal regulates the differentiation of Th1 cell and immune response in the lesion locations of $\mathrm{CD}$, thus increasing the release of Thl-type inflammatory cytokines IFN- $\gamma$ and TNF- $\alpha$ [39]. Study found that the gene-gene interactions between the SNPs of IL-12 and IL-12 receptor increase risk of developing some disease [40], and Il-12 can also induce tyrosine phosphorylation associated with the Il-12rb1 and then have multiple immunomodulatory effects [41]. IL-22 is a member of the IL-10 family of cytokines produced by adaptive and innate immune cells; it can also be produced by activated Th1 and Th17 cells. IL-22 exerts proinflammatory effects in IBD by acting on the colonic epithelial myofibroblasts to induce the expression of proinflammatory cytokines and matrix-degrading molecules [42]. The expression of IL-22 mRNA increased in both colon and mesenteric lymph nodes in mice with colitis [43]. Besides, the level of serum IL-22 is also significantly increased and correlated with disease activity [44]. IL-22RA2 is one of the receptors of IL-22; the expression of IL-22 mRNA and IL22RmRNA were both increased in inflammatory tissues [45]. 
IL-22 cannot be involved in the immunological activity of $B$ cells due to the absence of a functional IL-22R at the surface of these cells [46]. So, in combination with our results, we speculated that the expression trend of IL-12b, IL-22 and their receptors of IL-12rb1 and IL-12rb2 may be consistent.

Additionally, it is to be noted that normal saline was used as a control in the present study. In general, intragastric administration of normal saline has no effect on intestinal inflammation [47]. However, interestingly, we found that the body weight was increased and the gene expression was changed in CD with normal saline group rats, but the trend of improvement was lower than that of herb-partitioned moxibustion and mesalazine interventions. As moxibustion improved the pathological injury and inflammation of colon in $\mathrm{CD}$ rats while normal saline had no such obvious effect, we speculated that although saline solution altered gene expression through some ways unclear, it may not necessarily alter the translation process of genes and then take effect. Further studies can focus on protein expression of these genes after translation to reveal the unique regulating effect of moxibustion.

Pathway enrichment analysis indicated that pathways that involved differential genes such as the CAM pathway and the MAPK pathway were associated with inflammatory reactions [48-50]. However, autophagy-associated pathways were not enriched in this study. HTLV-I basic leucine zipper factor (HBZ) that exists in the human T-cell leukemia virus type1 (HTLV-1) infection pathway has been confirmed to regulate the activity of cellular transcription factors. In addition, HBZ can activate the mTOR mediated signaling pathways through binding to other factors [51]. In summary, herbpartitioned moxibustion might influence signaling pathways that are closely associated with CD through the regulation of autophagy- and immune-associated gene expression profiles in colon tissues of rats to effectively attenuate intestinal inflammation and promote the repair of intestinal tissue injury. These results will provide a new foundation for studies on the pathogenic mechanism of $\mathrm{CD}$ and the underlying mechanism of HPM treatment on CD from the perspectives of autophagy and immunology.

\section{Data Availability}

Data of partial gene sequencing have been presented in the article. The original data of excel format used to support the findings were supplied by $\mathrm{Lu}-\mathrm{Yi} \mathrm{Wu}$ under license and so cannot be made freely available. Requests for access to these data should be made to Lu-Yi Wu, luyitcm@163.com.

\section{Disclosure}

Ya-Nan Liu and Han-Dan Zheng are the co-first authors.

\section{Conflicts of Interest}

The authors declared that no conflicts of interest exist.

\section{Acknowledgments}

This research was supported by Shanghai Rising-Star Program, No. 16QA1403400; National Basic Research Program of China (973 Program), No. 2009CB522900 and No. 2015CB554501; National Natural Science Foundation of China, No. 81574079; "Dawn” Program of Shanghai Education Commission, No. 14SG39; and Program for Outstanding Medical Academic Leader (2015, No. 80). The authors thank the Beijing Genomics Institute (BGI) for the RNA high throughout sequencing and data analysis.

\section{Supplementary Materials}

The complementary information in the supplementary materials is as follows. Figure S1: body weights of rats in all groups at different time points. Figure S2: heatmap of the correlation between different groups. Figure S3: upregulation and downregulation of significantly differential genes between different groups. (Supplementary Materials)

\section{References}

[1] H. Z. Yang and Z. W. Hu, "The Immuno-regulatory and mechanisms of autophagy," International Journal of Immunology, vol. 33 , no. 3, pp. 165-168, 2010.

[2] T. Iida, K. Onodera, and H. Nakase, "Role of autophagy in the pathogenesis of inflammatory bowel disease," World Journal of Gastroenterology, vol. 23, no. 11, pp. 1944-1953, 2017.

[3] X. M. Wang, Z. Shi, M. P. Ma et al., "Thinking and methods of moxibustion regulation of pattern recognition receptors and signal transduction in inflammatory bowel disease," Shanghai Journal of Acupuncture and Moxibustion, vol. 29, no. 3, pp. 136139,2010

[4] H. G. Wu and L. S. Zhang, "Clinical study of herbs partition moxibustion on Crohns Disease," Modern Rehabilitation, vol. 4, no. 3 , article $397,2000$.

[5] C. Bao, L. Wu, H. Wu et al., "Moxibustion inhibits apoptosis and tumor necrosis factor-alpha/tumor necrosis factor receptor 1 in the colonic epithelium of crohn's disease model rats," Digestive Diseases and Sciences, vol. 57, no. 9, pp. 2286-2295, 2012.

[6] C.-H. Bao, L.-Y. Wu, Y. Shi et al., "Moxibustion down-regulates colonic epithelial cell apoptosis and repairs tight junctions in rats with Crohn's disease," World Journal of Gastroenterology, vol. 17, no. 45, pp. 4960-4970, 2011.

[7] G. P. Morris, P. L. Beck, M. S. Herridge, W. T. Depew, M. R. Szewczuk, and J. L. Wallace, "Hapten-induced model of chronic inflammation and ulceration in the rat colon," Gastroenterology, vol. 96, no. 3, pp. 795-803, 1989.

[8] Y. Guo, Experimental Acupuncture Science, China Press of Traditional Chinese Medicine, Beijing, China, 2008.

[9] H. J. Wang and L. X. Ji, "The location of the "Shenque" acupoint in rats," Acupuncture Research, vol. 32, no. 5, p. 312, 2007.

[10] C. R. Li, X. B. Hua, H. L. Zhou et al., "Development of acupuncture point map of guinea pigs," Shanghai Journal of Acupuncture and Moxibustion, pp. 28-30, 1992.

[11] S. Y. Xu, R. L. Ban, and X. Chen, Experimental Methodology of Pharmacology, People's Medical Publishing House, Beijing, China, 3rd edition, 2001. 
[12] F. Obermeier, N. Dunger, U. G. Strauch et al., "Contrasting activity of cytosine-guanosin dinucleotide oligonucleotides in mice with experimental colitis," Clinical \& Experimental Immunology, vol. 134, no. 2, pp. 217-224, 2003.

[13] Y. Shi and Wu. HG, "Clinical research of herb-partitioned moxibustion on the treatment of Crohns Disease," Jiangxi Journal of Traditional Chinese Medicine, vol. 34, no. 8, pp. 16-17, 2003.

[14] Y. Shi, C. H. Bao, H. G. Wu et al., "Effect of herbs-partitioned moxibustion on the expression of intestinal mucosa TNFa, TNFR1, TNFR2 and apoptosis of intestinal epithelial cells in Crohns disease patients," Shanghai Journal of Traditional Chinese Medicine, vol. 45, no. 1, pp. 46-50, 2011.

[15] C. H. Bao, J. M. Zhao, H. R. Liu et al., "Randomized controlled trial: moxibustion and acupuncture for the treatment of Crohn's disease," World Journal of Gastroenterology, vol. 20, no. 31, pp. 11000-11011, 2014.

[16] C. Bao, L. Wu, H. Wu et al., "Moxibustion inhibits apoptosis and tumor necrosis factor-alpha/tumor necrosis factor receptor 1 in the colonic epithelium of crohn's disease model rats," Digestive Diseases and Sciences, vol. 57, no. 9, pp. 2286-2295, 2012.

[17] D. C. Baumgart and S. R. Carding, "Inflammatory bowel disease: cause and immunobiology," The Lancet, vol. 369, no. 9573, pp. 1627-1640, 2007.

[18] M. Beaudoin, P. Goyette, G. Boucher et al., "Deep resequencing of GWAS loci identifies rare variants in CARD9, IL23R and RNF186 that are associated with ulcerative colitis," PLoS Genetics, vol. 9, no. 9, Article ID e1003723, 2013.

[19] A. Franke, D. P. McGovern, J. C. Barrett et al., "Genome-wide meta-analysis increases to 71 the number of confirmed Crohn's disease susceptibility loci," Nature Genetics, vol. 42, no. 12, pp. 1118-1125, 2010.

[20] J. P. Hugot, M. Chamaillard, H. Zouali et al., "Association of NOD2 leucine-rich repeat variants with susceptibility to Crohn's disease," Nature, vol. 411, no. 6837, pp. 599-603, 2001.

[21] Y. Ogura, D. K. Bonen, N. Inohara et al., "A frameshiftmutation in NOD2 associated with susceptibility to Crohn's disease," Nature, vol. 411, no. 6837, pp. 603-606, 2001.

[22] J. Hampe, A. Franke, P. Rosenstiel et al., "A genome-wide association scan of nonsynonymous SNPs identifies a susceptibility variant for Crohn disease in ATG16L1," Nature Genetics, vol. 39, no. 2, pp. 207-211, 2007.

[23] S. B. Singh, A. S. Davis, G. A. Taylor, and V. Deretic, "Human IRGM induces autophagy to eliminate intracellular mycobacteria," Science, vol. 313, no. 5792, pp. 1438-1441, 2006.

[24] L. Henckaerts, I. Cleynen, M. Brinar et al., "Genetic variation in the autophagy gene ULK1 and risk of Crohn's disease," Inflammatory Bowel Diseases, vol. 17, no. 6, pp. 1392-1397, 2011.

[25] C. R. Homer, A. L. Richmond, N. A. Rebert et al., "ATG16L1 and NOD2 interact in an autophagy-dependent antibacterial pathway implicated in Crohn's disease pathogenesis," Gastroenterology, vol. 139, no. 5, pp. 1630-1641, 2010.

[26] O. Brain, P. Allan, and A. Simmons, "NOD2-mediated autophagy and Crohn disease," Autophagy, vol. 6, no. 3, pp. 412414, 2010.

[27] M. Salem, M. Ammitzboell, K. Nys, J. B. Seidelin, and O. H. Nielsen, "ATG16L1: a multifunctional susceptibility factor in crohn disease," Autophagy, vol. 11, no. 4, pp. 585-594, 2015.

[28] K. Cadwell, J. Y. Liu, S. L. Brown et al., "A key role for autophagy and the autophagy gene Atg16l1 in mouse and human intestinal Paneth cells," Nature, vol. 456, no. 7219, pp. 259-263, 2008.
[29] M. T. Sorbara, L. K. Ellison, M. Ramjeet et al., "The protein ATG16L1 suppresses inflammatory cytokines induced by the intracellular sensors Nod1 and Nod2 in an autophagyindependent manner," Immunity, vol. 39, no. 5, pp. 858-873, 2013.

[30] N. Wang, H.-Y. Tan, S. Li, and Y. Feng, "Atg9b deficiency suppresses autophagy and potentiates endoplasmic reticulum stress-associated hepatocyte apoptosis in hepatocarcinogenesis," Theranostics, vol. 7, no. 8, pp. 2325-2338, 2017.

[31] S. Rufini, C. Ciccacci, D. Di Fusco et al., "Autophagy and inflammatory bowel disease: association between variants of the autophagy-related IRGM gene and susceptibility to Crohn's disease," Digestive and Liver Disease, vol. 47, no. 9, pp. 744-750, 2015.

[32] S. Chauhan, M. A. Mandell, and V. Deretic, "IRGM governs the core autophagy machinery to conduct antimicrobial defense," Molecular Cell, vol. 58, no. 3, pp. 507-521, 2015.

[33] S. Chauhan, M. A. Mandell, and V. Deretic, "Mechanism of action of the tuberculosis and Crohn disease risk factor IRGM in autophagy," Autophagy, vol. 12, no. 2, pp. 429-431, 2016.

[34] Y. Iwakura and H. Ishigame, "The IL-23/IL-17 axis in inflammation," The Journal of Clinical Investigation, vol. 116, no. 5, pp. 1218-1222, 2006.

[35] E. Bettelli, Y. Carrier, W. Gao et al., "Reciprocal developmental pathways for the generation of pathogenic effector TH17 and regulatory T cells," Nature, vol. 441, no. 7090, pp. 235-238, 2006.

[36] J. Seiderer, I. Elben, J. Diegelmann et al., "Role of the novel Th17 cytokine IL-17F in inflammatory bowel disease (IBD): Upregulated colonic IL-17F expression in active Crohn's disease and analysis of the IL17F p.His161Arg polymorphism in IBD," Inflammatory Bowel Diseases, vol. 14, no. 4, pp. 437-445, 2008.

[37] P. Behzadi, E. Behzadi, and R. Ranjbar, "Il-12 family cytokines: general characteristics, pathogenic microorganisms, receptors, and signalling pathways," Acta Microbiologica et Immunologica Hungarica, vol. 63, no. 1, pp. 1-25, 2016.

[38] D. A. Vignali and V. K. Kuchroo, "IL-12 family cytokines: immunological playmakers," Nature Immunology, vol. 13, no. 8 , pp. 722-728, 2012.

[39] T. Parrello, G Monteleone, S. Cucchiara et al., "Up-regulation of the IL-12 receptor b2 chain in Crohn's disease," Journal of Immunology, vol. 165, no. 12, pp. 7234-7239, 2000.

[40] J. Namkung, J. Lee, E. Kim et al., "Association of single nucleotide polymorphisms in the IL-12 (IL-12A and B) and IL12 receptor (IL-12R $\beta 1$ and $\beta 2$ ) genes and gene-gene interactions with atopic dermatitis in Koreans," Journal of Dermatological Science, vol. 57, no. 3, pp. 199-206, 2010.

[41] T. Kawashima, H. Kawasaki, T. Kitamura et al., "Interleukin12 induces tyrosine phosphorylation of an $85-\mathrm{kDa}$ protein associated with the interleukin-12 receptor beta 1 subunit," Cellular Immunology, vol. 186, no. 1, pp. 39-44, 1998.

[42] A. Andoh, Z. Zhang, O. Inatomi et al., "Interleukin-22, a member of the IL-10 subfamily, induces inflammatory responses in colonic subepithelial myofibroblasts," Gastroenterology, vol. 129, no. 3, pp. 969-984, 2005.

[43] K. Wolk, E. Witte, U. Hoffmann et al., "IL-22 induces lipopolysaccharide-binding protein in hepatocytes: a potential systemic role of IL-22 in Crohn's disease," The Journal of Immunology, vol. 178, no. 9, pp. 5973-5981, 2007.

[44] S. Schmechel, A. Konrad, J. Diegelmann et al., "Linking genetic susceptibility to Crohn's disease with Th17 cell function: IL-22 serum levels are increased in Crohn's disease and correlate with 
disease activity and IL23R genotype status," Inflammatory Bowel Diseases, vol. 14, no. 2, pp. 204-212, 2008.

[45] Z. Luo, H. Wang, Z. Sun, W. Luo, and Y. Wu, "Expression of IL22, IL-22R and IL-23 in the peri-implant soft tissues of patients with peri-implantitis," Archives of Oral Biolog, vol. 58, no. 5, pp. 523-529, 2013.

[46] S. Lécart, F. Morel, N. Noraz et al., "IL-22, in contrast to IL10 , does not induce Ig production, due to absence of a functional IL-22 receptor on activated human B cells," International Immunology, vol. 14, no. 11, pp. 1351-1356, 2002.

[47] J. Liu, W. Sun, X. Zhao et al., "Sanshensancao mixture alleviates the histologic injury and reduces the level of inflammatory factors of colonic mucosa in rats with ulcerative colitis," Chinese Journal of Cellular and Molecular Immunology, vol. 34, no. 10, pp. 876-879, 2018.

[48] T. Jayakumar, C. C. Chang, S. L. Lin et al., "Brazilin ameliorates high glucose-induced vascular inflammation via inhibiting ROS and CAMs production in human umbilical vein endothelial cells," BioMed Research International, vol. 2014, Article ID 403703, 10 pages, 2014.

[49] J.-L. Lai, Y.-H. Liu, C. Liu et al., "Indirubin inhibits LPS-induced inflammation via TLR4 abrogation mediated by the NF-kB and MAPK signaling pathways," Inflammation, vol. 40, no. 1, pp. 112, 2017.

[50] X.-N. Li, J. Su, L. Zhao et al., “The p38 MAPK inhibitor JLU1124 inhibits the inflammatory response induced by lipopolysaccharide through the MAPK-NF- $\kappa$ B pathway in RAW264.7 macrophages," International Immunopharmacology, vol. 17, no. 3, pp. 785-792, 2013.

[51] R. Mukai and T. Ohshima, "HTLV-1 HBZ positively regulates the mTOR signaling pathway via inhibition of GADD34 activity in the cytoplasm," Oncogene, vol. 33, no. 18, pp. 2317-2328, 2014. 


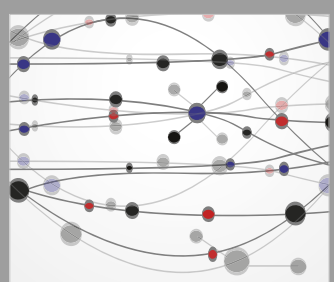

The Scientific World Journal
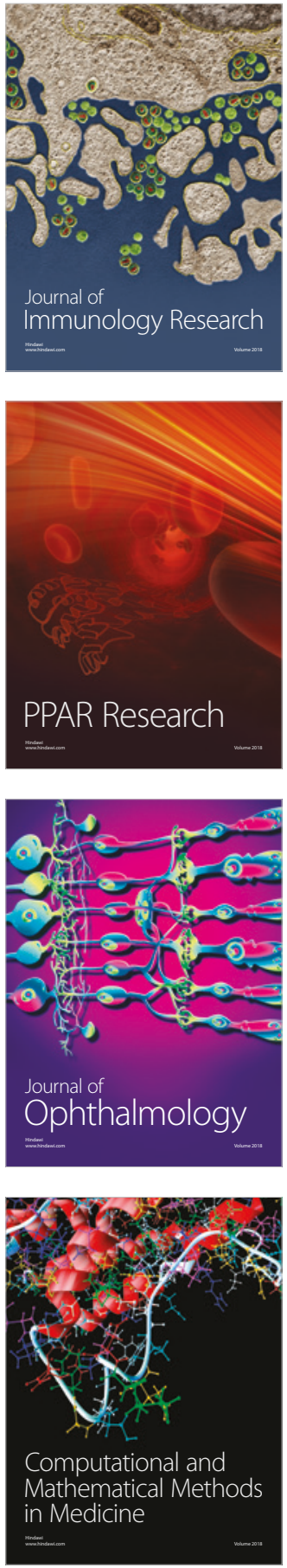

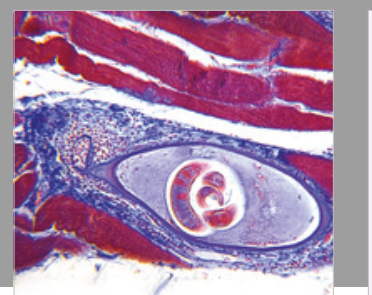

Gastroenterology Research and Practice

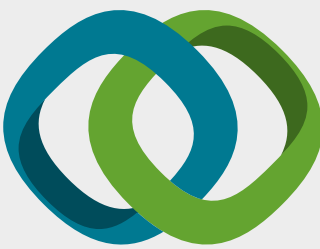

\section{Hindawi}

Submit your manuscripts at

www.hindawi.com
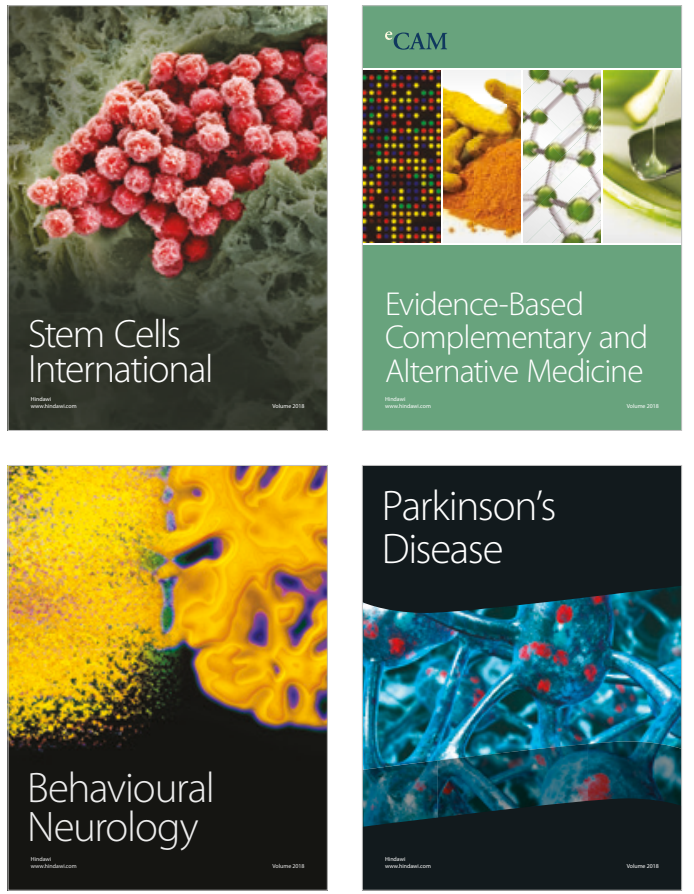

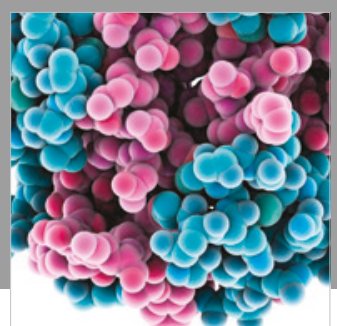

ournal of

Diabetes Research



Disease Markers
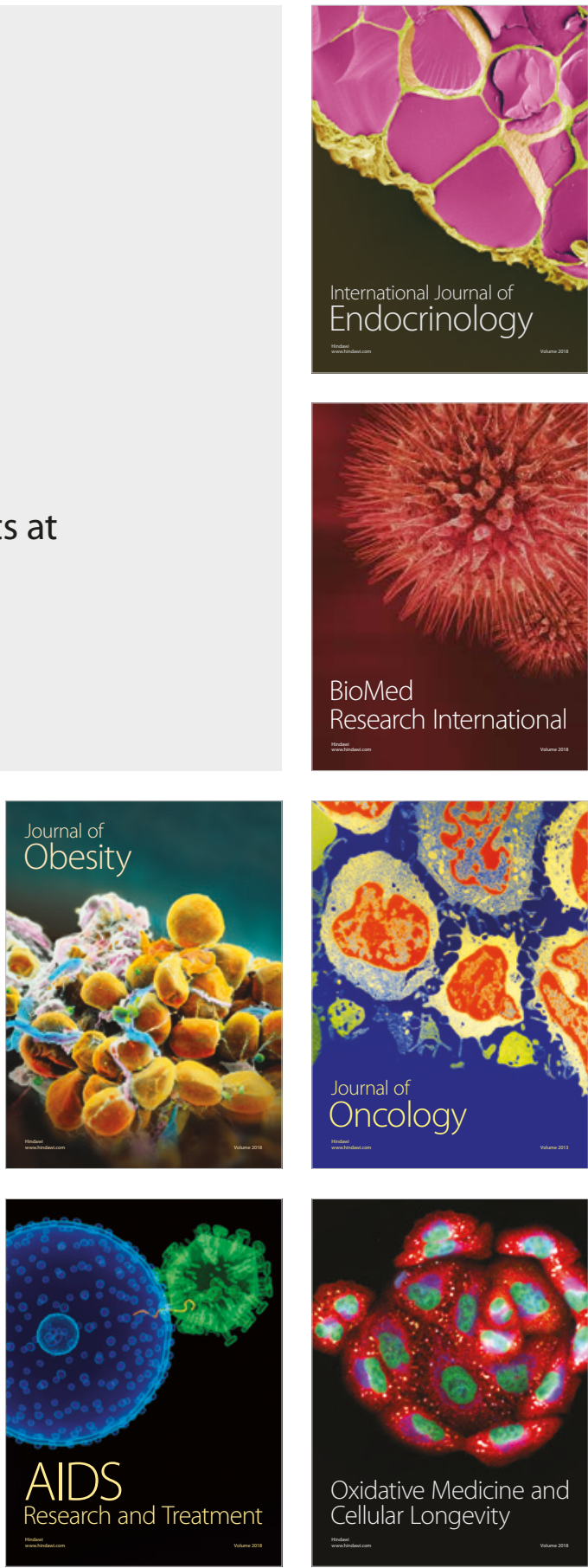